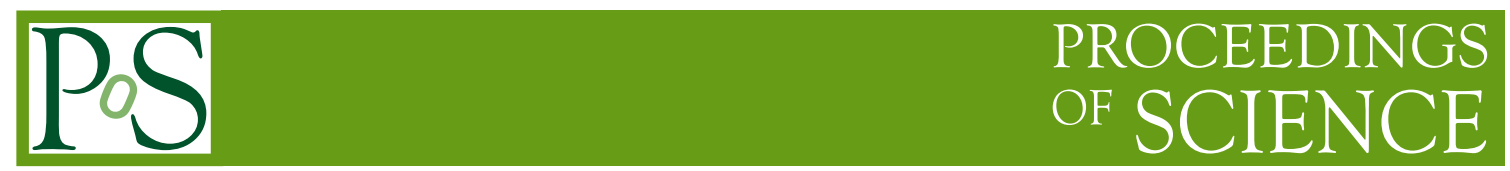

\title{
The Belle II SVD detector
}

K. R. Nakamura ${ }^{* p}$, K. Adamczyk ${ }^{r}$, H. Aihara ${ }^{o}$, C. Angelini ${ }^{h, i}$, T. Aziz ${ }^{g}$, V. Babu ${ }^{g}$,

S. Bacher ${ }^{r}$, S. Bahinipati ${ }^{d}$, E. Barberio ${ }^{a}$, Ti. Baroncelli $^{a}$, To. Baroncelli ${ }^{a}$,

A. K. Basith $^{e}$, G. Batignani ${ }^{h, i}$, A. Bauer ${ }^{b}$, P. K. Behera ${ }^{e}$, T. Bergauer ${ }^{b}$, S. Bettarini ${ }^{h, i}$,

B. Bhuyan ${ }^{f}$, T. Bilka ${ }^{c}$, F. Bosi ${ }^{i}$, L. Bosisio ${ }^{j, k}$, A. Bozek $^{r}$, F. Buchsteiner ${ }^{b}$, L. Bulla ${ }^{b}$,

G. Caria ${ }^{a}$, G. Casarosa ${ }^{i}$, M. Ceccanti $^{i}$, D. Červenkov ${ }^{c}$, S. R. Chendvankar ${ }^{g}$, N. Dash $^{d}$,

G. De Pietro ${ }^{h, i}$, S. T. Divekar ${ }^{g}$, Z. Doležal ${ }^{c}$, D. Dutta ${ }^{g}$, F. Forti $^{h, i}$, M. FriedI ${ }^{b}$, K. Hara $^{p}$,

T. Higuchi ${ }^{l}$, T. Horiguchi ${ }^{n}$, C. Irmler $^{b}$, A. Ishikawa ${ }^{n}$, H. B. Jeon ${ }^{q}$, C. Joo ${ }^{l}$, J. Kandra $^{c}$,

N. Kambara ${ }^{p}$, K. H. Kang ${ }^{q}$, T. Kawasaki ${ }^{c, m}$, P. Kodys ${ }^{c}$, T. Kohriki ${ }^{p}$, S. Koike ${ }^{D, p}$,

M. M. Kolwalkar ${ }^{g}$, R. Kumar ${ }^{s}$, W. Kun ${ }^{o}$, P. Kvasnička ${ }^{c}$, C. La Licata ${ }^{j, k}$, L. Lanceri ${ }^{j, k}$,

J. Lettenbicher ${ }^{b}$, J. Libby ${ }^{e}$, T. Lueck ${ }^{h, i}$, M. Maki $^{p}$, P. Mammini ${ }^{i}$, S. N. Mayekar ${ }^{g}$, $^{2}$

G. B. Mohanty ${ }^{g}$, S. Mohanty ${ }^{A, g}$, T. Morii ${ }^{l}$, Z. Natkaniec ${ }^{r}$, Y. Onuki ${ }^{o}$, W. Ostrowicz ${ }^{r}$,

A. Paladino ${ }^{h, i}$, E. Paoloni ${ }^{h, i}$, H. Park ${ }^{q}$, F. Pilo ${ }^{i}$, A. Profeti $^{i}$, I. Rashevskaya ${ }^{B, k}$,

K. K. Rao ${ }^{g}$, G. Rizzo ${ }^{h, i}$, Resmi P. K. ${ }^{e}$, M. Rozanska ${ }^{r}$, J. Sasaki ${ }^{o}$, N. Sato ${ }^{p}$,

S. Schultschik ${ }^{b}$, C. Schwanda ${ }^{b}$, Y. Seino ${ }^{m}$, N. Shimizu ${ }^{o}$, J. Stypula ${ }^{r}$, J. Suzuki $^{p}$, $^{2}$

S. Tanaka ${ }^{p}$, G. N. Taylor ${ }^{a}$, R. Thalmeier ${ }^{b}$, R. Thomas ${ }^{g}$, T. Tsuboyama ${ }^{p}$, S. Uozumi ${ }^{q}$,

P. Urquijo ${ }^{a}$, L. Vitale ${ }^{j, k}$, S. Watanuki ${ }^{n}$, M. Watanabe ${ }^{l}$, I. J. Watson ${ }^{o}$, J. Webb $^{a}$,

J. Wiechczynski $^{r}$, S. Williams ${ }^{a}$, B. Würkner ${ }^{b}$, H. Yamamoto ${ }^{n}$, H. Yin ${ }^{b}$, T. Yoshinobu ${ }^{p}$, and L. Zani ${ }^{h, i}$

(Belle II SVD Collaboration) 
${ }^{a}$ School of Physics, University of Melbourne, Melbourne, Victoria 3010, Australia

${ }^{b}$ Institute of High Energy Physics, Austrian Academy of Sciences, 1050 Vienna, Austria

${ }^{c}$ Faculty of Mathematics and Physics, Charles University, 12116 Prague, Czech Republic

${ }^{d}$ Indian Institute of Technology Bhubaneswar, Satya Nagar, India

${ }^{e}$ Indian Institute of Technology Madras, Chennai 600036, India

${ }^{f}$ Indian Institute of Technology Guwahati, Assam 781039, India

${ }^{g}$ Tata Institute of Fundamental Research, Mumbai 400005, India, ${ }^{A}$ also at Utkal University, Bhubaneswar 751004, India

${ }^{h}$ Dipartimento di Fisica, Università di Pisa, I-56127 Pisa, Italy

${ }^{i}$ INFN Sezione di Pisa, I-56127 Pisa, Italy

${ }^{j}$ Dipartimento di Fisica, Università di Trieste, I-34127 Trieste, Italy

${ }^{k}$ INFN Sezione di Trieste, I-34127 Trieste, Italy, ${ }^{B}$ presently at TIFPA - INFN, I-38123 Trento, Italy

${ }^{l}$ Kavli Institute for the Physics and Mathematics of the Universe (WPI), University of Tokyo, Kashiwa 277-8583, Japan

${ }^{m}$ Department of Physics, Niigata University, Niigata 950-2181, Japan, ${ }^{C}$ presently at Kitasato University, Sagamihara 252-0373, Japan

${ }^{n}$ Department of Physics, Tohoku University, Sendai 980-8578, Japan

${ }^{o}$ Department of Physics, University of Tokyo, Tokyo 113-0033, Japan

${ }^{p}$ High Energy Accelerator Research Organization (KEK), Tsukuba 305-0801, Japan, ${ }^{D}$ deceased

${ }^{q}$ Department of Physics, Kyungpook National University, Daegu 702-701, Korea

${ }^{r}$ H. Niewodniczanski Institute of Nuclear Physics, Krakow 31-342, Poland

${ }^{s}$ Punjab Agricultural University, Ludhiana 141004, India

E-mail: katsuro.nakamuradkek.jp

The Silicon Vertex Detector (SVD) is one of the main detectors in the Belle II experiment at KEK, Japan. In combination with a pixel detector, the SVD determines precise decay vertices and performs low-momentum track reconstruction. The SVD ladders are being developed at several institutes. For the development of the tracking algorithm as well as the performance estimation of the ladders, beam tests for the ladders were performed. We report an overview of the SVD development, its performance measured in the beam tests, and the prospect of its assembly and commissioning until installation.

The 25th International workshop on vertex detectors

September 26-30, 2016

La Biodola, Isola d' Elba, ITALY

${ }^{*}$ Speaker. 


\section{Overview of SVD detector}

The Belle II experiment [ [W] is an intensity frontier experiment whose main goal is to discover physics beyond the standard model (BSM) by indirect means. The experiment is installed at an interaction point of the SuperKEKB [వ] collider at KEK (Tsukuba, Japan), which is an $e^{+} e^{-}$ collider operating at a center-of-mass energy near the $\Upsilon(4 S)$ resonance. The $e^{+}$and $e^{-}$beams in SuperKEKB have energies of 4 and $7 \mathrm{GeV}$, respectively, with a beam crossing angle of $41.5 \mathrm{mrad}$. The collider is designed to have an instantaneous luminosity of $L=8.0 \times 10^{35} \mathrm{~cm}^{-2} \mathrm{~s}^{-1}$, which is 40 times larger than the previous KEKB collider for the Belle experiment. The detectors and accelerator are being developed for the start of physics experiment in 2018.

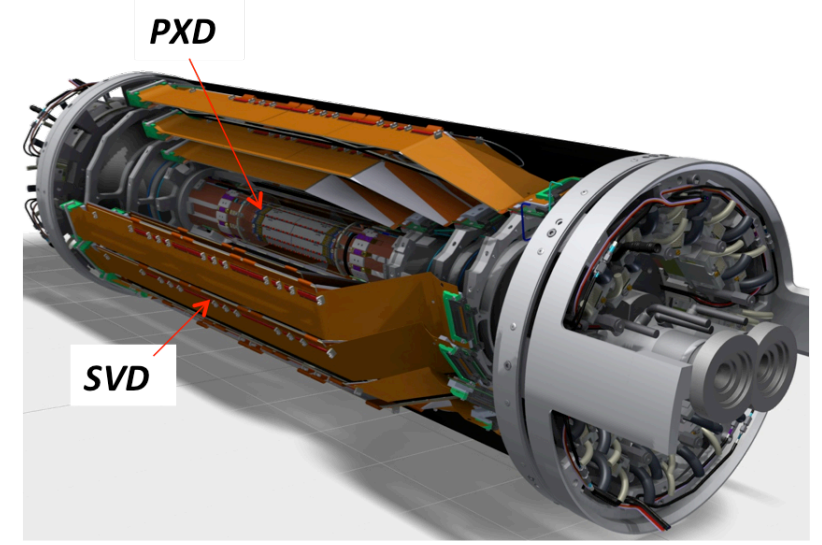

Figure 1: The Belle II Vertex Detector (VXD), which consists of the PXD and the SVD.

In the Belle II experiment, precise determination of the decay vertex position and low-momentum tracking are essential for the search of BSM physics, in particular to look for possible BSM contributions to the $\mathrm{CP}$ violation asymmetry in the beauty and charm sector. These tasks are performed by two silicon detectors: PiXel Detector (PXD) and Silicon Vertex Detector (SVD). The combination of these two detectors together goes by the name of the VerteX Detector (VXD). Figure $\square$ shows the 3D CAD model of the VXD. The PXD consists of DEPFET [B] pixel sensors that form the inner two layers of the VXD, while the SVD comprises the outer four layers, that are equipped with Double-sided Silicon Strip Detectors (DSSD). Going from inside to outside, the four SVD layers are named Layer-3, 4, 5, and 6. In order to operate in the high beam background of SuperKEKB [䧃], a short shaping time on the front-end electronics, a radiation hardness of more than $100 \mathrm{kGy}$ are required on the SVD. Moreover, the SVD must have standalone tracking capability down to a transverse momentum of $50 \mathrm{MeV} / c$.

The SVD consists of ladders which are arrays of DSSD modules. Layer-3, 4, 5, and 6 have $7,10,12$, and 16 ladders cylindrically arranged around the interaction point. Figure $\square$ shows a cross-sectional view of the VXD and the SVD ladders. The Layer-3 ladder is straight, whereas in Layer-4, 5, and 6 the last DSSD is slanted under 11.9, 16.0, and 21.1 degree angles, respectively. The purposes of the slanted shape is to reduce overall material budget and the number of sensors, and at the same time to improve the hit quality by avoiding shallow hits with large cluster widths. The radial distance from the interaction point of the DSSD sensors are 39, 80, 115, and $140 \mathrm{~mm}$ 


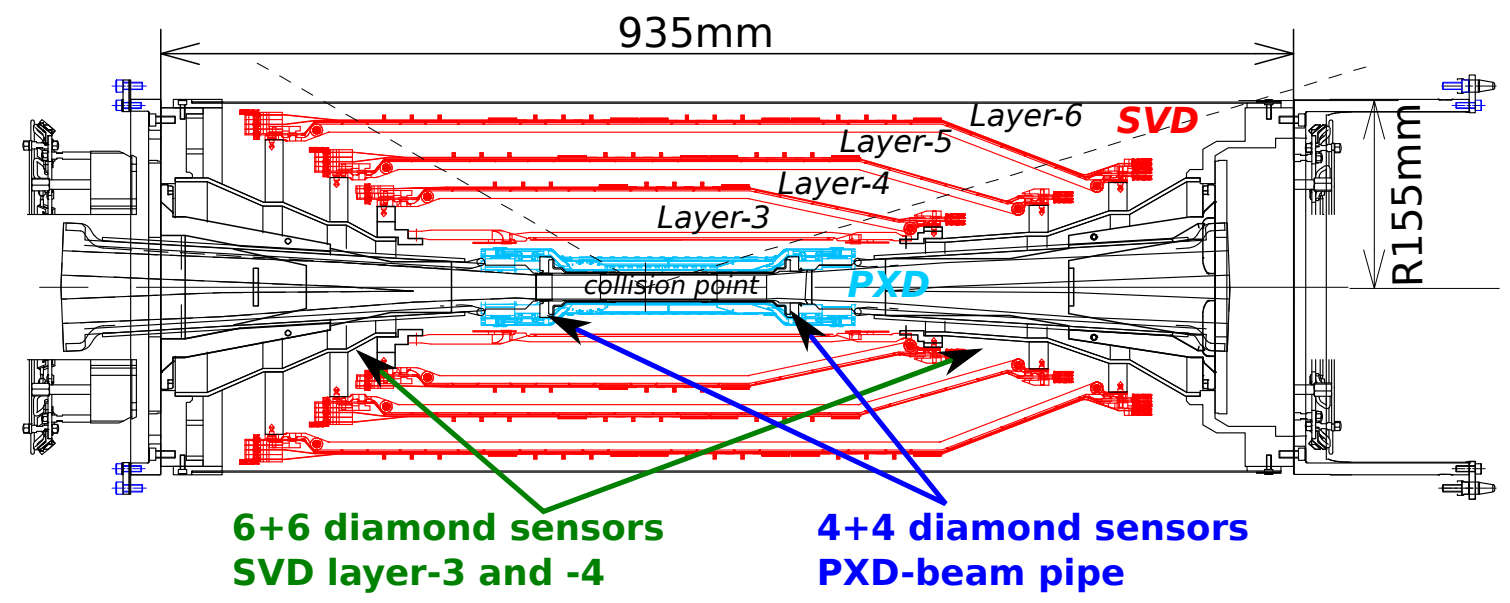

Figure 2: The Belle II VXD which consists of the PXD (blue ladders) and SVD (red ladders). Locations of diamond sensors for beam background monitor are also shown.

for Layer-3, 4, 5, and 6, respectively. The average material budget of each layer is about $0.7 \%$ of the radiation length including stainless cooling pipes for the dual-phase $\mathrm{CO}_{2}$ cooling.

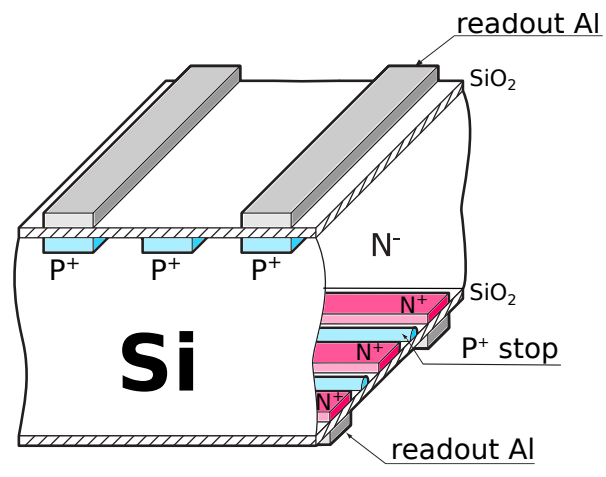

Figure 3: A cross section image of the DSSD sensor.

There are three types of the DSSD sensor, large and small rectangular sensors made by Hamamatsu Photonics K.K. (HPK) and trapezoidal ones from Micron. The rectangular and trapezoidal sensors have thicknesses of 320 and $300 \mu \mathrm{m}$, respectively. P-strips in the sensor are in the longitudinal direction (same as the beam direction) and $\mathrm{n}$-strips are in the transverse direction. The readout strip pitches in p-strips are 75 (50) $\mu \mathrm{m}$ for the large (small) rectangular sensors, and 50-75 $\mu \mathrm{m}$ for the trapezoidal sensors. The pitches in n-strips are $240 \mu \mathrm{m}$ for the large rectangular and trapezoidal sensors, and $160 \mu \mathrm{m}$ for the small rectangular sensors. A cross section image of the DSSD sensor is shown in Fig. [1]. The sensor has intermediate strips without readout aluminum channels in both p- and n-strips.

The front-end readout ASIC of the SVD is the APV25 chip [5], originally developed for the CMS silicon tracking detector. The chip has a short shaping time of $50 \mathrm{~ns}$ and a good radiation hardness of over 1 MGy. Each APV25 has 128 read-out channels and dissipates a maximum of $0.4 \mathrm{~W}$. In total, 1748 APV25 chips are implemented on the SVD.

In order to minimize the analog path length and hence capacitive noise, APV25 chips for the 
DSSDs are to be located as close as possible to the sensors. For the DSSDs on both ends of the ladders (forward and backward), APV25 chips are located on the far end of the sensor, outside of the detector acceptance. On the other hand, for the intermediate sensors, APV25 chips are to be located on the sensors. A flexible circuit, which is named "ORIGAMI flex", is glued on the sensor with an electrical and thermal isolation foam $\left(\right.$ AIREX $\left.^{\circledR}\right)$, and APV25 chips are mounted on the ORIGAMI flex. Because these chips are within the detector acceptance, they are thinned down to $100 \mu \mathrm{m}$ to reduce the material budget.

The heat dissipation from the APV25 chips on the SVD is absorbed with dual-phase $\mathrm{CO}_{2}$ cooling system [目]. Thin stainless tubes with an outer diameter of $1.6 \mathrm{~mm}$ and a thickness of $0.1 \mathrm{~mm}$ are brought into thermal contact with the APV25 chips with thermal-conductive sheets (Softtherm ${ }^{\circledR} 86 / 125$ ). The liquid-gas mixture $\mathrm{CO}_{2}$ coolant with a temperature of about $-20{ }^{\circ} \mathrm{C}$ flows inside these tubes.

\section{SVD ladder assembly procedure and schedule}

The ladder assembly is performed by several institutes. The University of Melbourne (Australia) assembles Layer-3 ladders. At the INFN-University of Pisa (Italy), forward and backward subassemblies for Layer 4-6 are assembled. The subassemblies are shipped to HEPHY (Austria) and Kavli IPMU (Japan). At HEPHY Layer-5 ladders are assembled, while at Kavli IPMU Layer4 and Layer-6 ladders are assembled (Layer-4 ladders are the responsibility of the TIFR (India) members). The ladders assembled at all assembly sites are critically reviewed by all other group members ensuring the qualities of the assembled ladders are uniform. All the assembled ladders are finally shipped to KEK where the SVD will be finally installed.

In all ladder assembly sites, the following quality assurance tests are performed for the assembled ladders. To check the mechanical precision, shifts of the DSSD sensors from the designed coordinates in XYZ direction are measured with an optical coordinate measurement machine (CMM). Typically the shifts are less than $150 \mu \mathrm{m}$. As a confirmation of the DSSD sensor functionality, the $I-V$ curve is measured. To check for possible defects and to verify the overall DSSD performance, signal readout test of the ladders is performed with either laser pulse injection or $\beta$-source $\mathrm{Sr}^{90}$.

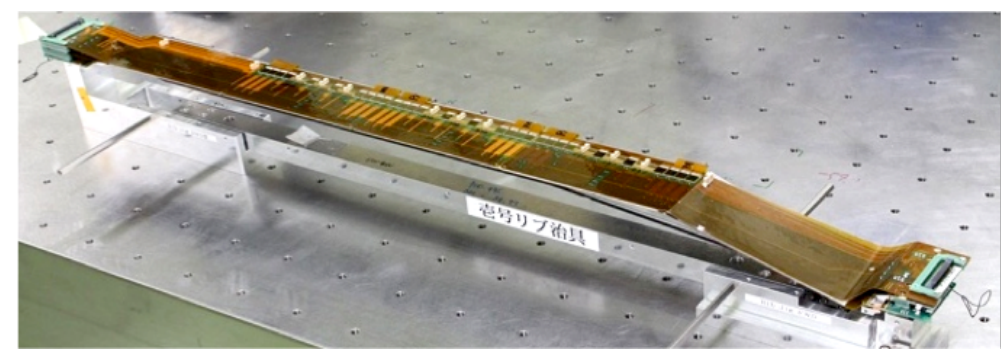

Figure 4: A produced Layer-6 ladder.

The mass production of ladders was started early 2016. Figure 1 shows a fully assembled and qualified Layer-6 ladder. The completion of the production is scheduled on November 2017.

The SVD integration at KEK is the final step to complete the SVD construction. The integration procedure must be safe and well established, as possible mistake can destroy all mounted 
ladders at once. Now tools for a safe SVD integration are being developed. Prototypes of all necessary assembly tools have been produced. The preliminary procedure was reviewed by a reviewcommittee including external members. We are planning to finalize the tools by February 2017 and consequently start the SVD integration. We start the SVD integration before the completion of ladder production as we expect to have enough number of ladders produced by that time.

The SVD construction is scheduled to be completed by December 2017. We will then start integration of the Belle II beam pipe, PXD, and SVD to produce the VXD. We plan to install the VXD into the Belle II detector around June 2018 for the Belle II physics running to start during the last quarter of 2018.

\section{SVD performance study}

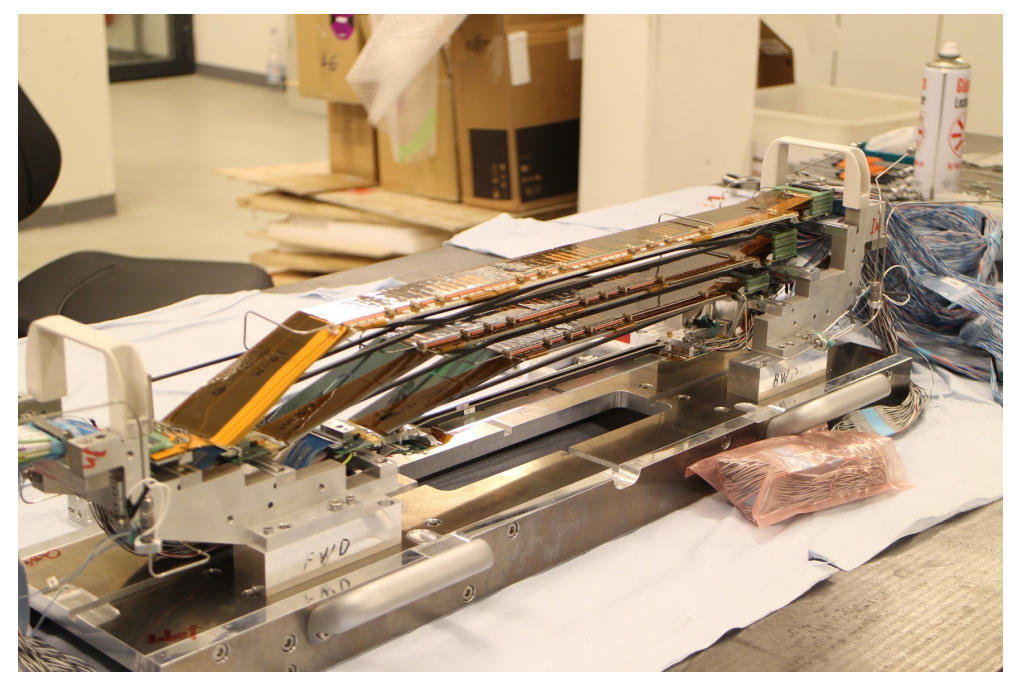

Figure 5: Setup of the SVD ladders in DESY beam test during January 2016. Ladders from all four layers are used in the setup.

Performance study for the SVD ladders was performed in tests with an electron beam and a charged-hadron beam $[\square]$. The charged-hadron beam test was performed at CERN during June 2015 with $120 \mathrm{GeV}$ beam for study of Layer-5 ladder performance. The electron beam test was at DESY during April 2016 with 2-5 GeV electron beam for study of ladders of all four layers. The collision energy loss $(d E / d x)$ of these $2-5 \mathrm{GeV}$ electrons in the silicon sensor are about $40 \%$ larger than the one of MIP particles. Figure $\square$ shows the SVD setup in this beam test. The four ladders are aligned along a same direction and located at the same radial locations as the final geometry. We combined the SVD setup with the 2-layers PXD prototype sensors and tested the VXD tracking performance as well. This was the first test for the VXD tracking with the real SVD ladders.

The Signal-to-Noise Ratio (SNR) is defined as the cluster charge divided by the noise value of the strip. If the cluster width is more than 1 , the noise value is estimated as the sum in quadrature of the noises of clusterized strips. Figure 6 shows the SNR distribution of a Layer-5 ladder tested at a hadron beam with an energy of $120 \mathrm{GeV}$. These plots also show the distributions of cluster widths 1, 2 and more than 2. The listed numbers in the plots are the most probable SNR values of these distributions. 


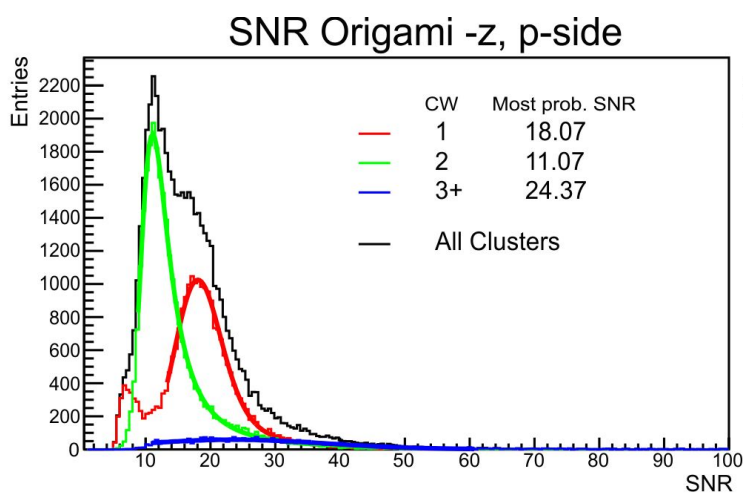

(a) p-strip side

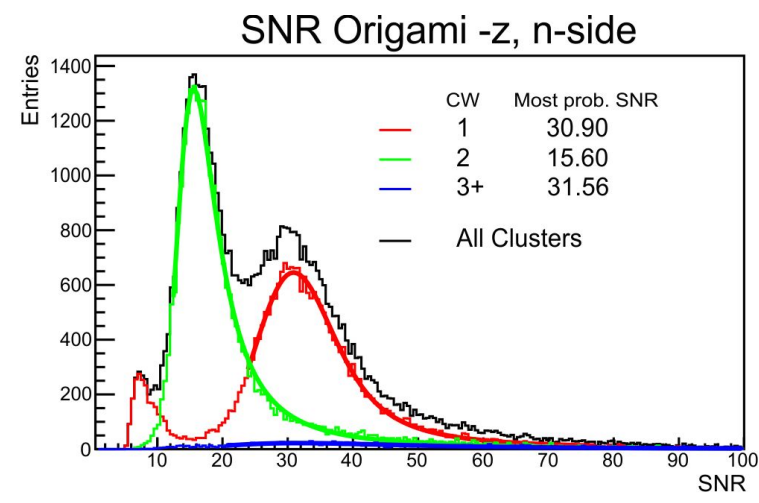

(b) n-strip side

Figure 6: SNR distributions of a tested DSSD in (a) p-strips and (b) n-strips without cooling for a perpendicularly incident charged hadron beam with an energy of $120 \mathrm{GeV}$. (O-Z in the Layer-5 ladder). Histograms for cluster widths (CWs) of 1, 2, and more than 2 are shown in red, green, and blue, respectively, with fitted curves of Landau functions (convoluted with Gaussian). The most probable SNR values for these cluster widths are also shown.

Charges created by tracks traversing the sensor at the position of a read-out strip are collected on that strip and produce clusters with cluster width 1 . As the charge collection efficiency of the readout strip is almost $100 \%$, the obtained signals of cluster width 1 are similar in $\mathrm{p}$ - and n-strips. The noise values in n-strips are smaller than those in p-strips due to a shorter $n$-strip length. Hence, for cluster width $1, \mathrm{n}$-strips have larger SNR values than $\mathrm{p}$-strips.

Charges created by tracks traversing the sensor at the position of an intermediate strip without readout are collected on the neighboring strips and produce clusters with cluster width 2. Capacitive coupling between the strips and backplane of the sensor causes charge-collection loss on the intermediate strips, because this coupling to backplane is comparable to the one to neighboring readout strips [ []$]$. The loss in charge collection is larger in n-strips (about 25\% loss for large HPK sensor ${ }^{1}$ ) compared to $\mathrm{p}$-strips (about $10 \%$ loss for large HPK sensor) because of smaller coupling to neighboring strips in n-side due to a wider pitch. Therefore, the ratio between the most probable SNR values of cluster width 1 and 2 are smaller than $1 / \sqrt{2}$. We confirmed that the SNR values in both $\mathrm{p}$ - and n-strips are well above 10 even for a cluster width of 2 .

\footnotetext{
${ }^{1}$ The loss is calculated with capacitance values estimated from the strip geometry.
} 
Table 1: The most probable SNR values of cluster width 1 before and after $\mathrm{CO}_{2}$ cooling $\left(-20{ }^{\circ} \mathrm{C}\right)$ for a perpendicularly incident charged hadron beam with an energy of $120 \mathrm{GeV}$.

\begin{tabular}{rccc}
\hline Most prob. SNR & before cooling & after cooling & ratio \\
\hline \hline p-strip side & 18.1 & 21.1 & 1.17 \\
n-strip side & 30.9 & 35.1 & 1.14 \\
\hline
\end{tabular}

The $\mathrm{CO}_{2}$ cooling improves the SNR distribution because it cools down the APV25 chips reducing thermal noises on the chips. The resulting SNR values of the Landau peaks for the clusters width of 1 before and after the $\mathrm{CO}_{2}$ cooling is listed in Table $\mathrm{W}$. In this test, the $\mathrm{CO}_{2}$ coolant temperature was $-20{ }^{\circ} \mathrm{C}$. We observed more than $10 \%$ improvement in SNR due to the cooling.

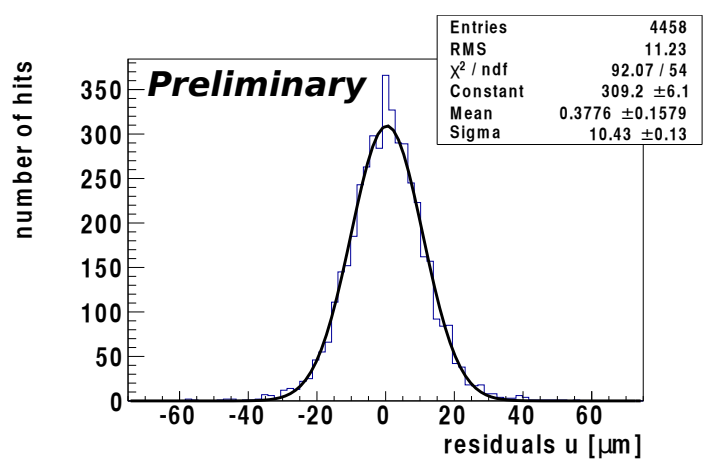

(a) p-strip side

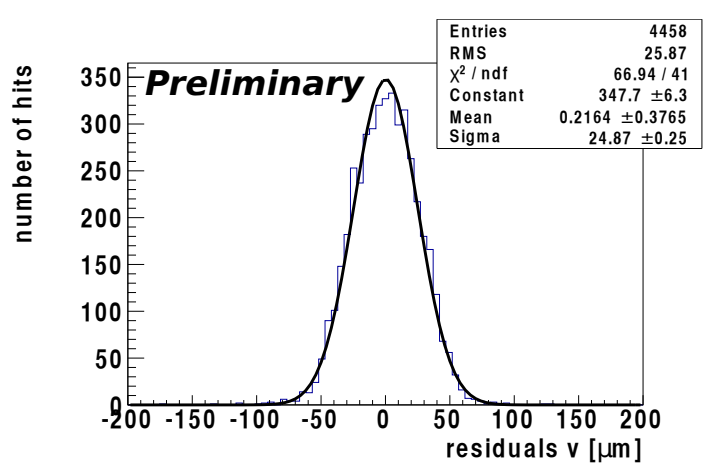

(b) n-strip side

Figure 7: Cluster residual distribution in (a) p-strips and (b) n-strips of the Layer-3 ladder.

In the DESY beam test, we reconstructed electron tracks using the clusterized hits on the four ladders. In order to check the position resolution of the ladder in each layer, we studied distributions of cluster residuals that are differences of the cluster hit positions from projected positions of tracks reconstructed with other layers. Figure $\mathbb{Z}$ shows the residual distributions in the Layer-3 ladder. We fitted the distributions with Gaussian functions. The fitted widths of the Gaussian are $10.4 \mu \mathrm{m}$ in p-strips and $24.9 \mu \mathrm{m}$ in $\mathrm{n}$-strips. From simulation, track extrapolation uncertainties of $\sim 7 \mu \mathrm{m}$ in p-strips and $\sim 8 \mu \mathrm{m}$ in $\mathrm{n}$-strips are estimated. Considering these uncertainties, DSSD position resolutions are estimated to be $8.2 \mu \mathrm{m}$ in p-strips and $23.6 \mu \mathrm{m}$ in n-strips. These results are consistent with our estimations from the strip pitch, $p / 2 \sqrt{12}$, where the factor 2 comes from intermediate strips.

For the next study, we calculated the efficiency of the DSSD cluster hit for the tracks. In each position along the track projection, we derived probabilities that the cluster hits associated to the tracks reconstructed with other layers. The resulting efficiencies in the Layer-5 ladder are plotted on Fig. [8. The empty areas in the plot are known noisy channels which were masked out in the analysis and boundary of neighboring sensors. We confirmed excellent efficiencies of higher than $99 \%$ in all the layers. 


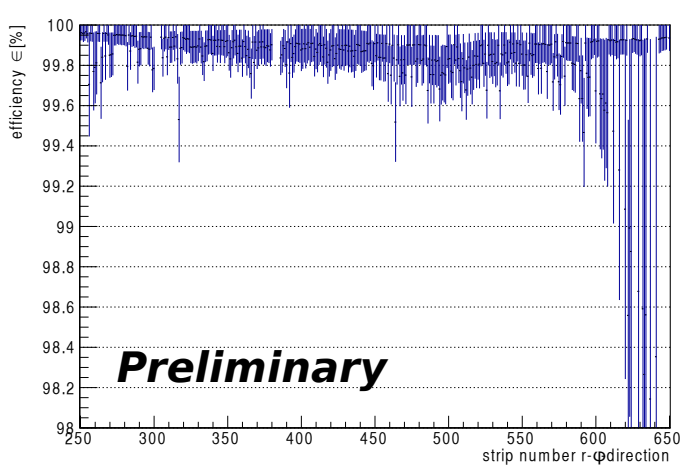

(a) p-strip side

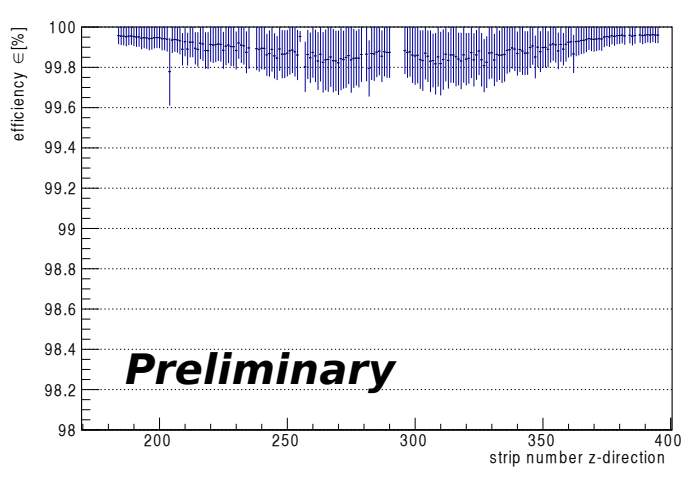

(b) n-strip side

Figure 8: DSSD hit efficiencies in (a) p-strips and (b) n-strips of the Layer-5 ladder as functions of trackprojected positions.

\section{Radiation monitoring for beam abort}

An accidental beam background enhancement can damage the SVD. Hence the beam background must be monitored during the experiment and whenever the background gets high the beam must be immediately dumped. For that purpose, we install diamond detectors in the VXD volume.

The detector uses a single crystal diamond of dimension $4.5 \times 4.5 \times 0.5 \mathrm{~mm}^{3}$, made via a chemical vapor deposition (CVD). The crystal has double-sided metallization made of Ti(100 nm) $\operatorname{Pt}(120 \mathrm{~nm})-\operatorname{Au}(250 \mathrm{~nm})$. The crystal is held in a package of dimension $12 \times 20 \times 3.1 \mathrm{~mm}^{3}$. We measure current on the bias line of the diamond with long high-quality cables.

The diamond detector has a number of merits for the beam background monitor. It has high radiation tolerance and good timing resolution. Moreover, the temperature dependence of signal gain in the diamond detector is small. The detector structure is rather simple and compact.

A pair of 4 diamond detectors are installed on two locations of the beam pipe around both ends of the PXD, and a pair of 6 such detectors are installed on two locations of the SVD support structure. These locations are shown in Fig. \. In total, 20 diamond detectors are deployed in the VXD volume. In each location, the diamond detector surrounds the beam pipe isotropically so that angular distribution of the beam background can be detected.

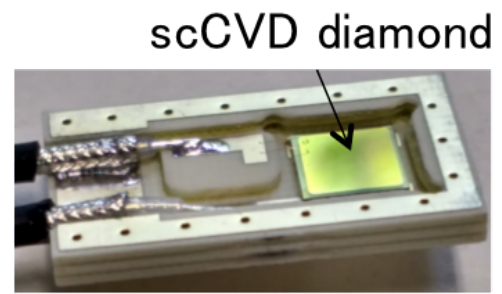

Figure 9: Prototype of the diamond detector (inside the package).

Prototypes of the diamond detector (Fig. Q) were produced. They were tested on the SuperKEKB beam lines during SuperKEKB phase-1 commissioning without beam collisions. Figure $\square$ shows the detector currents in different beam size setting. With decreasing the beam size, 


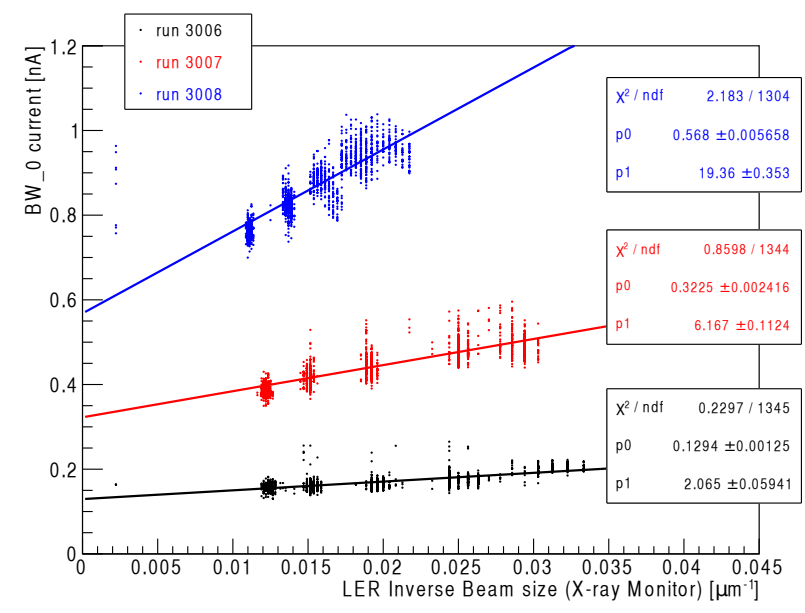

Figure 10: Detector currents in the diamond detector as a function of the beam size measured when SuperKEKB $e^{+}$beam was stored on the beam line. Three data sets in different beam currents, $160 \mathrm{~mA}$ (black), $360 \mathrm{~mA}$ (red), and $540 \mathrm{~mA}$ (blue), are plotted.

we can see a systematic enhancement on the detector current due to increase of the Touschek beam background. We confirmed that the diamond detector works well as a beam background monitor.

\section{Conclusions}

The SVD is an essential detector for the Belle II experiment for precise vertex determination and low-momentum track reconstruction. It consists of four-layers of DSSD ladders. The ladder mass-production in all assembly sites has started since early 2016. The production is planned to be over by November 2017. The SVD integration with the produced ladders will be performed at KEK and be completed by December 2017.

We studied the SVD performance, especially the ladder SNR, position resolution, and cluster hit efficiency. These results show an excellent performance of the SVD with a SNR of greater than 10 , position resolutions consistent with expectation, cluster hit efficiency of more than $99 \%$.

The diamond detector for the beam background monitor in the VXD volume is also being developed. The prototype is produced and tested on the SuperKEKB beam line during the phase-1 commissioning. The result shows good sensitivity for the beam background.

\section{References}

[1] T. Abe, et al., KEK Report 2010-1, arXiv1011.352v1

[2] Y. Ohnishi, et al., Prog. Theor. Exp. Phys. (2013) 03A011

[3] T. Abe, et al., KEK Report 2010-1 (2010) [arXiv:1011.0352]

[4] H. Nakayama, et al., Proceedings of HF2014, Beijing, China, FRT3A1 (2014), 110

[5] M. J. French, et al., Nucl. Instr. and Meth. A466 (2011), 359-365

[6] B. Verlaat and A. Colijn, PoS VERTEX2009 (2009) 031 
[7] C. Irmler, et al., JINST 11 (2015) C01087

176

[8] M. Krammer and H. Pernegger, Nucl. Instr. and Meth. A397 (1997) 232-242 